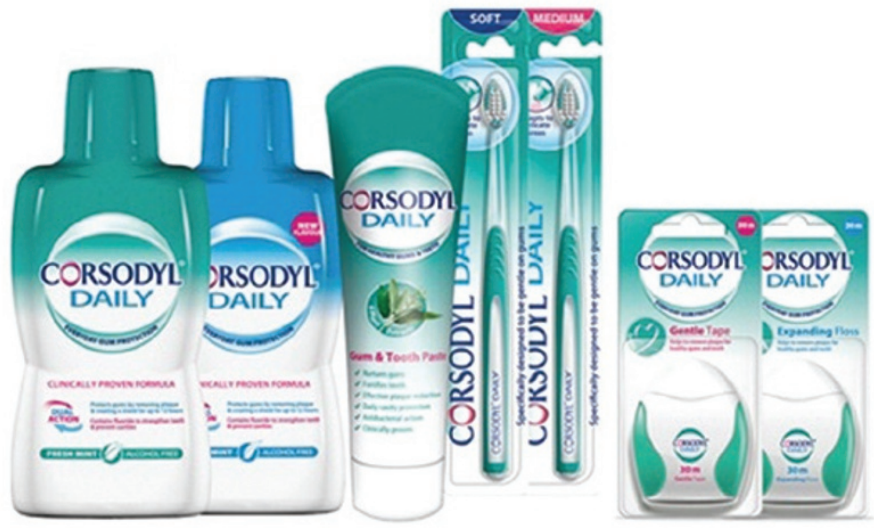

\section{FOUR NEW PRODUCTS FOR CORSODYL}

Corsodyl has announced the launch of four new products within the Corsodyl Daily range to help patients remove plaque on a daily basis and maintain healthy gums.

The extended range includes two new interdental cleaning products: Corsodyl Daily Expanding Floss, which expands to fill interproximal spaces while flossing to help remove plaque and debris between teeth, and Corsodyl Daily Gentle Tape to provide gentle interdental cleaning.

The new Corsodyl Daily toothbrushes are available in soft or medium bristles, and have small dome shaped heads to clean even hard to reach areas effectively. The shorter outer bristles of the brushes adapt to delicate areas, and are specifically designed to be gentle on gums.

The Corsodyl Daily mouthwash packaging and claims have been updated, and a new 'Cool Mint' flavour has been added which contains the same 250 ppm fluoride and $0.06 \%$ chlorhexidine digluconate as the Fresh Mint formulation. Both Corsodyl Daily mouthwashes are alcohol free. In conjunction with Corsodyl Daily Gum \& Tooth Paste, these new products make it easier than ever to recommend the Corsodyl Daily range as a complete oral care routine for the maintenance of healthy gums. wuw.corsodyl.co.uk

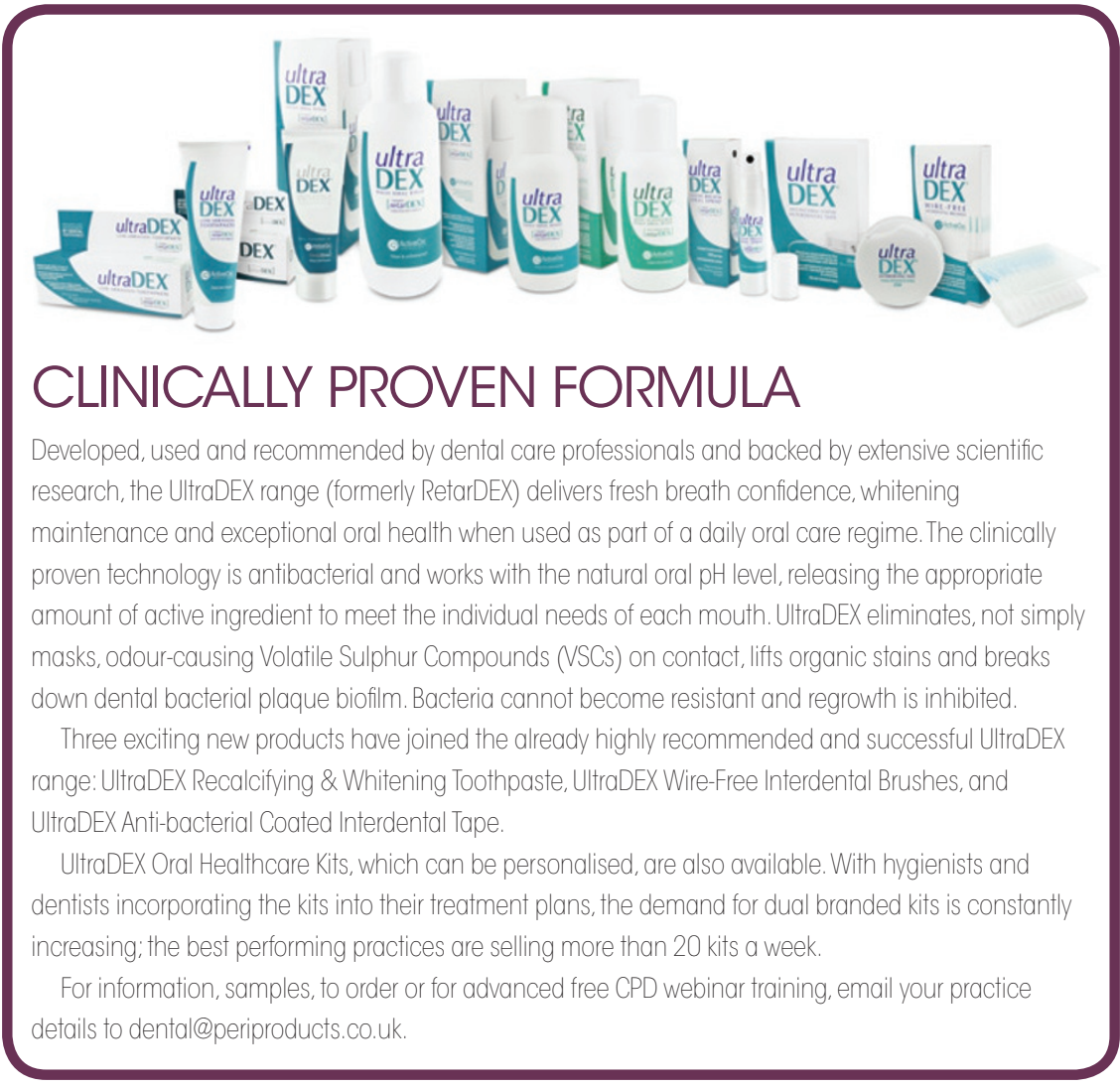

DENTINE HYPERSENSITIVITY AFFECTS 1 IN 3

The Sensodyne team is committed to raising awareness and understanding of dentine hypersensitivity, as patients may be unaware of the factors which might predispose to the condition, or that simple interventions could help relieve their sensitivity.

Help inform your patients with the new patient information pieces from Sensodyne, available now for your practice. The new pieces include an A4 tent card for use chairside, and a patient questionnaire with display stand for use at reception. Both materials offer information on dentine hypersensitivity, and on how a toothpaste such as Sensodyne Repair \& Protect could help.

You can review condition information and order trial sized packs of selected Sensodyne products at www.gskdentalprofessionals.co.uk.

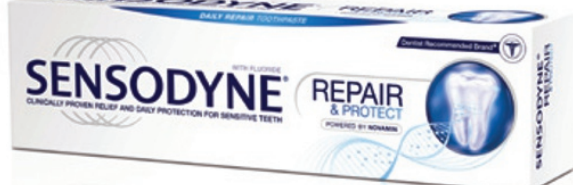

If you would like to promote your products or services direct to the dental industry through VITAL UPDATE telephone Andy on 02078434785 or Lyle on 02078434724. 